\title{
Intra-Articular Injection of an Extracellular Vesicle Isolate Product to Treat Knee Osteoarthritis in an Athlete
}

\author{
Johnny East DO' ${ }^{1}$, Maxwell Dordevic ${ }^{*}$
}

\author{
${ }^{1}$ Addison Pain \& Regenerative Medicine, 16633 Dallas Pkwy Suite 150, USA
}

\begin{abstract}
The patient is a 51 years old athlete with a $2 \frac{1 / 2}{y r}$. history of increasing left medial and patella-femoral knee pain. He had previously failed two arthroscopic procedures, a hyaluronic acid and PRP injections. MRI and arthroscopy indicated Kellgren-Lawrence grade three arthritis in the medial femoraltibial and patellofemoral areas of the knee joint. He underwent a single injection of bone marrow-derived mesenchymal stem cell EVIP (ExoFlo Direct Biologics, St. Louis MO) containing active growth factors (over 800) and exosomes (0ver 10 Billion per cc). Within two weeks he had returned to normal activities. At six-month follow-up he feels his overall improvement is $75 \%$. His improvement at six-month follow-up in BPI was $96 \%$, ODI $84 \%$ and LEFS $59 \%$. There were no complications with the injection.
\end{abstract}

Keywords: Osteoarthritis, Arthroscopic, Arthroplasty.

\section{Introduction}

Knee osteoarthritis (OA) has been demonstrated in cadaver and radiographic studies to affect as many as 55 million patients over the age of 60 [1]. Patients with knee OA have pain, crepitus, loss of motion, and decreased ability to bear weight or ambulate. Limiting the ability to ambulate severely impairs the activities of daily living. Nonsurgical treatments for knee OA according to the American Academy of Orthopedic Surgeons (AAOS) include weight loss, gentle exercise, and the use of non-steroidal anti-inflammatory medications (NSAIDs). The surgical treatment for knee OA is total knee arthroplasty (TKA) [2]. The AAOS does not recommend knee arthroscopy or the use of any Hyaluronic Acid injections (HLA) [2].

Over the last several years it has become increasingly understood by researchers and clinicians that the clinical efficacy of utilizing mesenchymal stem cells (MSCs) to treat osteoarthritis (OA) is not dependent on the cells differentiating into articular cartilage, rather, it is reliant entirely upon their paracrine release of growth factors (GFs) and exosomes. Living MSCs are not required to accomplish the release of GFs and exosomes into an arthritic joint $[12,13]$. This case report will introduce the concept of using an acellular MSC derived EVIP containing active growth factors (over 800) and exosomes (over 10 Billion per cc) to treat knee OA and the rationale of why acellular therapies will replace all current cellular biologic therapies both autogenous and allogeneic.

\section{Materials and Methods}

This is a case report of an EVIP injection for the treatment of knee osteoarthritis. OA is defined by swelling, pain, and stiffness in the knee joint that is worsened by weight-bearing and ambulation. Radiographs and MRI scanning are used to grade osteoarthritis of the tibial-femoral joint from one to four using the Kellgen-Lawrence scale [6].

The patient is a 51 years old male athlete. $\mathrm{He}$ presented on $23^{\text {rd }}$ July, 2019 with complaints of increasing medial left knee pain. He initially injured the left knee in January 2017 in a bicycle accident. Within a few weeks, he underwent an arthroscopic lateral meniscectomy. He continued to complain of increasing medial knee pain.

He underwent a second arthroscopic procedure on $13^{\text {th }}$ July, 2018 for a partial medial meniscectomy, tricompartmental extensive synovectomy, and chondroplasty for grade three patellofemoral and medial tibiofemoral arthritis.

Corresponding Author: Maxwell Dordevic, Addison Pain \& Regenerative Medicine, 16633 Dallas Pkwy Suite 150, USA Received date: January 06, 2020; Accepted date: January 15, 2020; Published date: January 16, 2020.

DOI: https://doi.org/10.31546/JBRCI.1005 
MRI and radiographs revealed Kellgren-Lawrence grade four changes of the medial and patellofemoral compartments. Pictures from the left knee arthroscopy are shown in Figure one. Following the second arthroscopic procedure he continued to experience left knee pain exacerbated by activities and ambulation. During November of 2018 he had three hyaluronic acid injections, a platelet rich plasma (PRP) injection, and physical therapy.

He presented to our clinic on $23^{\text {rd }}$ July,2019 with complaints of increasing left knee pain exacerbated with ambulation and activities. Examination of the left knee showed a ROM of $0^{0}$ $120^{\circ}$, stable ligaments in varus, valgus, AP stress tests, pain to palpation in the medial, and patella-femoral joints. He had a 1+ effusion. The patient was evaluated with a Brief Pain Inventory (BPI), a Lower Extremity Functional Index (LEFI), and an Oswestry Disability Index (ODI) prior to the injection of the EVIP, 6 weeks and at 3-month follow-up.
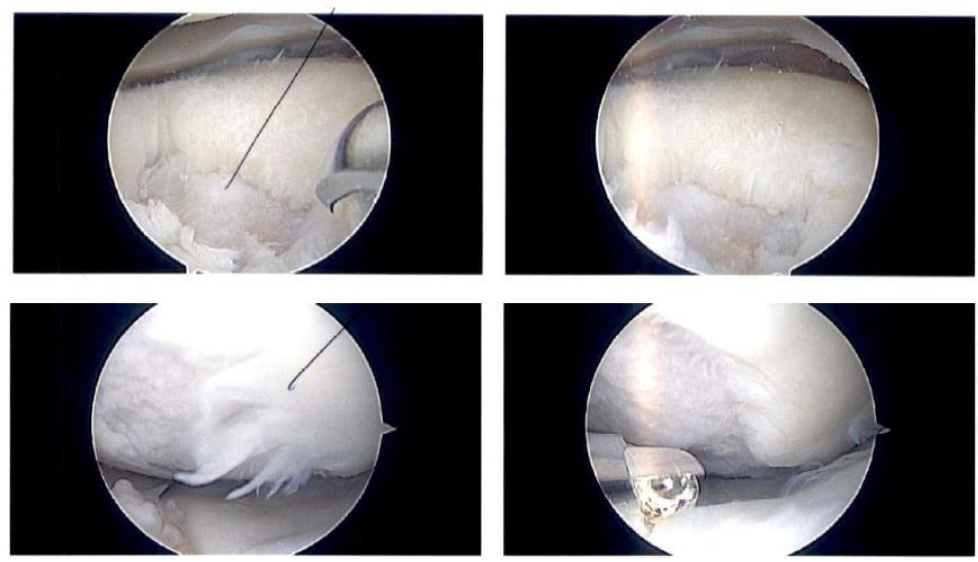

Figure 1: Knee arthroscopy showed Kellgren-Lawrence Grade four arthritis of the medial tibial femoral joint.

\section{The Knee Injection}

After counseling and consent he agreed to undergo an injection of frozen bone marrow-derived mesenchymal stem cell EVIP (ExoFlo-Direct Biologics, St. Louis, MO) containing active growth factors and exosomes.

The left knee was sterilized with betadine skin prep. A 20gauge needle was placed through a lateral para-patellar approach into the knee joint. Needle placement was verified by fluoroscopy. At this point, $2 \mathrm{cc}$ of the frozen EVIP (ExoFloDirect Biologics, St. Louis MO) was thawed to room temperature and placed into the joint.

\section{Clinical Results:}

The patient was put on restricted physical activity for 1 week following the procedure. Passive low-resistance range of motion was encouraged immediately. The patient returned to full activities at two weeks.
The patient's clinical results are detailed in Figure two and Table one. A lower Brief Pain Inventory and Oswestry Disability Index score is preferred. A higher Lower Extremity Functional Scale is preferred. The patient self-reported to opine his knee was about $75 \%$ improved from 4 weeks to 12 weeks after the injection. The patient will continue to be followed for two years after the procedure.

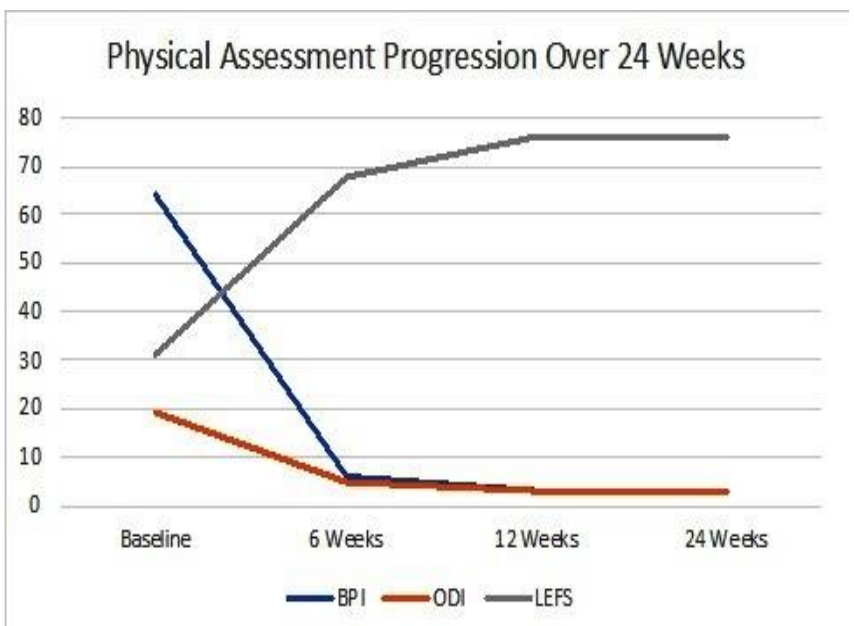

Figure 2: A lower BPI and ODI=improvement, a higher LEFS score=improvement

Table 1: Physical evaluation measurements with the percent improvement over the initial 12 weeks.

\begin{tabular}{|l|c|c|c|c|c|}
\hline & Baseline & 6 Weeks & 12 Weeks & 24 Weeks & Percent Improvement \\
\hline BPI & 64 & 6 & 3 & 3 & $96 \%$ \\
\hline ODI & 19 & 5 & 3 & 3 & $84 \%$ \\
\hline LEFS & 31 & 68 & 76 & 76 & $59 \%$ \\
\hline
\end{tabular}

\section{Discussion}

The patient is a 51 years old athlete with a $2 \frac{1 / 2}{\mathrm{yr}}$. history of increasing left medial and patella-femoral knee pain. He had previously failed two arthroscopic procedures, a hyalouronic acid and PRP injections. MRI and arthroscopy indicated Kellgren-Lawrence grade three arthritis in the medial femoraltibial and patellofemoal areas of the knee joint.

He underwent a single injection of bone marrowderived mesenchymal stem cell EVIP (ExoFlo Direct Biologics, St. Louis MO) containing active growth factors (over 800) and exosomes (0ver 10 Billion per cc). Within two weeks he had returned to normal activities. At six-month follow-up he feels his overall improvement is $75 \%$. 
His improvement at six-month follow-up in BPI was 96\%, ODI $84 \%$, and LEFS $59 \%$. There was no change in improvement between three month and six month follow up. There were no complications with the injection.

The knee is a diarthrodial joint with a synovial lining and a joint capsule. The synovial capsule contains numerous synovial MSCs (more than found in bone marrow or adipose). These MSCs have more chondrogenic potential than bone or adipose MSCs [14-17]. During the development of OA, proinflammatory growth factors are produced by these synovial MSCs. This creates a chronically inflamed, painful joint environment. Bone marrow concentrate (BMC) contains on average only about 2,500 MSCs per cc. Despite the incredibly small number of MSCs found in BMC; there is extensive literature reporting clinical efficacy in animals and humans using BMC for the treatment of OA.[3-5,7-11] This effect cannot be dependent upon BMC/MSC cell survival or differentiation. The efficacious effect must be from the release of acellular paracrine factors. The future of the biologic treatment of OA will be the utilization of acellular MSC derived growth factors, especially exosomes. The exosome is a tiny 30 to 150 nanometer-sized (1 billionth of a meter) biphospholipid membrane-enclosed structure created by the Golgi body or apparatus.

An MSC (12 to 18 microns) is 1,000 times larger than an exosome. The diameter of a hair is 80,000 nanometers. Exosomes contain growth factors, signaling lipids and micro and messenger RNA. The RNA contents in exosomes mediate most of their anti-inflammatory effects. The RNA is placed into an exosome along with numerous peptide growth factors. These paracrine factors can be placed into any joint in concentrations of 100,000 or more times that of any cellular MSC treatment. These growth factor proteins and exosomes will function in a paracrine fashion to both, directly and indirectly, alter the inflammatory environment of any painful arthritic joint back to a normal non-painful physiologic environment. Figure 3 diagrams this process.

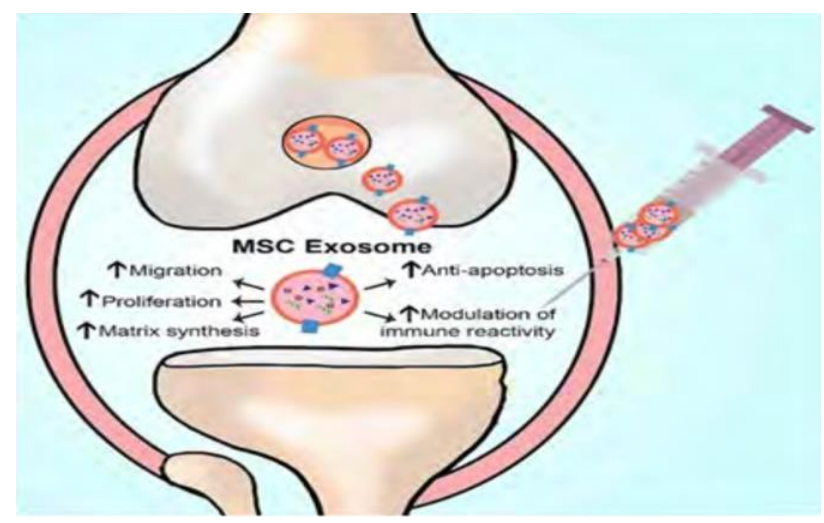

Figure 3: Diagram of the MSC Exosome treatment for OA.
The future acellular treatment for OA will involve a two-front attack. First, highly concentrated antiinflammatory MSC derived growth factors are injected into the arthritic joint. These growth factors will enter the nucleus of the recipient synovial MSC. The EVIP growth factors will stimulate transcription of mRNA containing instructions for the production of continuous anti-inflammatory secretomes, chemokines, and cytokines. These will be released from the recipient synovial MSC into the synovial fluid. Second, the highly concentrated exosomes from the EVIP will enter recipient synovial MSCs to deliver their mRNA. This delivered mRNA will directly undergo translation in the recipient synovial MSC ribosomes to produce anti-inflammatory secretomes, cytokines, and chemokines. These salubrious effects could last months or years. This acellular biologic treatment can all be achieved with a single arthritic joint injection, not requiring the morbidity and cost of obtaining autogenous MSCs. The future of regenerative medicine in orthopedics and spine may well be the utilization of highly concentrated acellular MSC derived growth factors and especially exosomes [7-11].

Consent for Publication: Written informed consent was obtained from the patient for the publication of this case report.

Conflicts of Interest: The authors declare no conflict of interest.

\section{References:}

1. Osteoarthritis health center-knee osteoarthritis. Webmd: Osteoarthritis of the knee.

2. Michael J Wiater, Stuart J Fischer. Arthritis of the knee - American Academy of Orthopedic Surgeons. 2013.

3. Black LL, Gaynor J, Adams C, et al. Effect of intraarticular injection of autologous adiposederived mesenchymal stem and regenerative cells on clinical signs of chronic osteoarthritis of the elbow joint in dogs. Vet Ther. 2008; 9:192-200.

4. Murphy JM, Fink DJ, Hunziker EB, et al. Stem cell therapy in a caprine model of osteoarthritis. Arthritis Rheum. 2003; 48:3464-3474.

5. Lee KB, Hui JH, Song IC, et al. Injectable mesenchymal stem cell therapy for large cartilage defects-a porcine model. Stem Cells. 2007; 25:2964-2971. 
6. Kellgren J, Lawrence J. Radiological assessment of OsteoArthrosis. Ann Rheum Dis. 1957; 16:494-502.

7. Wang A, Feng Y, Yu H. Application of Mesenchymal Stem Cell Therapy for the Treatment of Osteo Arthritis- A Concise Review. World J Stem Cells. 2019; 11:222-235.

8. Li Z, Wang Y, Xiao K, Weng X. Emerging Role of Exosomes in the Joint Diseases. Cell Physiol Biochem. 2018; 47:2008-2017.

9. Chang Y, Wu K, Harn H. Exosomes and Stem Cells in Degenerative Disease Diagnosis and Therapy. Cell Transplant. 2018; 27:349-363.

10. Cheng L, Zhang K, Wu S, et al. Focus on Mesenchymal Stem Cell-Derived Exosomes: Opportunities and Challenges in Cell-Free Therapy. Stem Cells Int. 2017;2017:6305295.

11. Zhang S, Chuah SJ, Lai RC, et.al. MSC Exosomes Mediate Cartilage Repair by Enhancing Proliferation, Attenuating Apoptosis and Modulating Immune Reactivity. Biomaterials. 2018; 156:16-27.

12. Caplan AI, Correa D. The MSC: An injury drugstore. Cell Stem Cell. 2011; 9:11-15.

13. Caplan AI, Dennis JE. Mesenchymal stem cells as trophic mediators. J Cell Biochem. 2006; 98:1076-1084.

14. Pettine KA, Murphy MB, Suzuki RK, Sand TT. Percutaneous injection of Autologous bone marrow concentrate significantly reduces lumbar discogenic pain through twelve months. Stem Cells. 2015; 33:146-156.

15. Pettine K, Dordevic M. The Biologic Treatment of Osteoarthritis with Mesenchymal Stem Cell Exosomes: The Future is Now. J Stem Cell Res Dev Ther. 2019; 5:S1001.

16. Koga H, Muneta $\mathrm{T}, \mathrm{Ju} \mathrm{YJ}$, et al. Synovial Stem cells are Regionally Specified According to Local Microenvironments after Implantation for Cartilage Regeneration. Stem Cells. 2007; 25:689-696.

17. Fan J, Varshney RR, Ren L, et al. Synovium-Derived Mesenchymal stem cells: A new source for musculoskeletal regeneration. Tissue Eng Part B Rev. 2009 ; 15:75-86. 